\title{
Risk Factors for Periprosthetic Joint Infections Following Primary Total Hip Arthroplasty
}

\author{
Jesenko Michael ${ }^{1, *}$, Windhager Reinhard ${ }^{1}$, Kontekakis Antonis ${ }^{2}$, Hanstein Tim $^{2}$, Kuehn Klaus-Dieter ${ }^{1}$ \\ ${ }^{1}$ Vienna General Hospital, Department of Orthopedics, Austria \\ ${ }^{2}$ Heraeus Medical GmbH, Germany
}

Copyright $(\mathcal{C} 2016$ by authors, all rights reserved. Authors agree that this article remains permanently open access under the terms of the Creative Commons Attribution License 4.0 International License

\begin{abstract}
Total hip arthroplasty (THA) is a very successful and effective operation for the restoration of the hip function. The number of operations is steadily climbing and is going to reach new heights in the future. The most feared and devastating complication is the periprosthetic joint infection (PJI) and has in most cases to be treated with a total revision of the prostheses. The risk factors for a PJI are playing a very important role in the preoperative assessment of the patient and for the type of antibiotic treatment. There are many risk factors described in the literature, which are associated with the development of a PJI. Our goal was to analyze and find the relevant risk factors, which matter most in joint replacement surgery. We searched the database "PubMed" and "Embase" with the keywords: „((hip AND infection) AND (arthroplasty OR replacement))". With the help of check lists and limits we extracted the most viable studies for our research. Risk factors associated with a deep periprosthetic joint infection included the BMI (Body mass index), male gender, prolonged duration of surgery, diabetes mellitus type 2, the ASA (American society of anesthesiologists) score, the Charlson score and the NNIS (National Nosocomial Infections Surveillance System) risk index score. Female gender, age and the diagnosis of rheumatoid arthritis were not associated with a higher risk of periprosthetic joint infections. Patients with risk factors should be assessed and informed preoperatively and should receive an appropriate prophylactic antibiotic treatment, to reduce the risk of a PJI. With the reduction of their weight and adjustment of the diabetes, patients can reduce the risk for PJI by their own. Total hip replacement is still a very safe and effective operation and therefore should not be withheld from patients.
\end{abstract}

Keywords THA, Infection, PJI, Risk Factors, BMI, Diabetes, Gender, Age, Operation Time

\section{Introduction}

The total hip arthroplasty evolved from a difficult and expensive operation to a very cost efficient restoration of the hip function and treatment of pain. Meanwhile more than one million THAs are performed per year worldwide.[1] Through the rising number of operations the number of complications are increasing too. Periprosthetic joint infections (PJI) is by far the most devastating and severe complication with an incidence between 0.5 and $3 \%$ in primary surgery. [2]

Infections cause a large financial burden for the healthcare system. While a primary total hip arthroplasty in Germany costs $6.263 €$ in average, the expenses rise to $29.331 €$ when the patient develops a PJI. Most of the costs are due to a prolonged stay in the hospital and thereof resulting staff costs. [3] Many studies tried to find the predisposing factors for developing an infection, but it is hard to say, which factors are really crucial and which are not. The results seem also very different and partially contrary. The goal of this study was to find published studies regarding the deep periprosthetic infection, to compare and evaluate their results. Through these we would be able to reduce the number of infections and save many patients from suffering pain. 
Table 1. Risk factors (plus sign) and no risk factors (minus sign) sorted by number of patients.

\begin{tabular}{|c|c|c|c|c|c|c|c|c|c|c|c|}
\hline & E & $\frac{\oplus}{\sqrt{\frac{10}{\Sigma}}}$ & 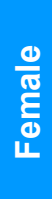 & 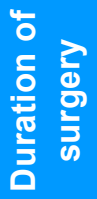 & $\begin{array}{l}\text { d } \\
\text { d] } \\
\frac{0}{10} \\
\frac{0}{0}\end{array}$ & 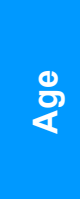 & ब & $\frac{\infty}{z}$ & $\begin{array}{l}\frac{5}{0} \\
\frac{\text { 을 }}{\frac{\pi}{0}} \\
\frac{1}{0}\end{array}$ & 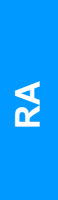 & 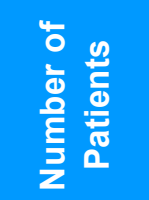 \\
\hline $\begin{array}{c}\text { Dale, Fenstad } \\
2012\end{array}$ & & & - & & & - & & & & + & 432.168 \\
\hline $\begin{array}{c}\text { Pedersen, } \\
\text { Svendsson } 2010\end{array}$ & & + & - & + & & & & & + & - & 80.756 \\
\hline Dale, Hallan 2009 & & + & - & + & & - & & & & - & 79.820 \\
\hline Pedersen 2010 & & & & & + & & & & & & 57.575 \\
\hline Bozic, Lau 2012 & + & & & & & & & & & + & 40.919 \\
\hline Ong 2009 & & + & - & + & & & & & + & & 39.929 \\
\hline Namba 2012 & + & - & + & - & - & - & + & & & & 30.491 \\
\hline Muilwijk 2006 & & & & + & & + & + & + & & & 26.127 \\
\hline Lubekke 2007 & + & & & & & & & & & & 2.186 \\
\hline Reina 2013 & + & + & - & & & & + & & & & 2.107 \\
\hline Davis 2011 & - & & & & & & & & & & 1.617 \\
\hline Andrew 2008 & - & & & & & & & & & & 1.356 \\
\hline Moran 2005 & - & & & & & & & & & & 759 \\
\hline Bozic 2014 & + & + & - & & & & & & & - & 587 \\
\hline $\begin{array}{c}\text { Font-Vizcarra } \\
2011\end{array}$ & & & & & & + & & & & & 400 \\
\hline & + & Risk f & & & - & no ris & ctor & & & & \\
\hline
\end{tabular}




\section{Materials and Methods}

We conducted a search with the term: "((hip AND infection) AND (arthroplasty OR replacement))" using the databases PubMed and Embase. The results of the databases were compared with the program "Reference Manager", the doubly present studies were sorted out and transferred in a "Microsoft Excel" sheet for further investigation. The studies were evaluated with a previous determined checklist to assure a consistency in the selection process. The decision on difficult cases and disagreements were made after consulting with experts to discuss their exclusion and prevent any mistakes in the selection process. The excluded studies were listed in the Excel sheet with their reasons of exclusion. Only original studies were used, while systematic reviews and meta-analyses were further investigated for their original studies and their possibility of inclusion. The studies were screened with the following criteria:

1. Language: The Language must be English or German, since these languages are available in the involved departments.

2. Inappropriate study type: Animal studies, in-vitro studies, comments and editorials must be excluded, since these studies will not provide additional knowledge regarding risk factors for the general population.

3. Site other than hip: Studies not regarding the hip arthroplasty have to be excluded.

4. Outcome not hip infection: The outcome of the study has to be the hip infection since other complications are not the goal of this study.

5. Not a risk factor study: If the study looks at the outcome of interest, but does not report risk factors for an infection of the site of the hip, it has to be excluded.
6. Not a patient related risk factor: The study reports risk factors for the outcome of interest, but those are not patient related risk factors (e.g. hospital volume, drug efficacy)

7. Full text not available: The full text of the study has to be available for our staff.

8. Combined outcome: If studies report risk factors in combination with another site (e.g. knee) since the combination could bias the results.

9. Not primary THA: Our study looks only at primary THAs since the combination with other procedures could bias the results.

10. Outcome not a PJI: If the study fulfills all the criteria above, but reports only superficial hip infection or a combination of superficial hip infection with PJI it has to be excluded.

\section{Results}

Our search provided a total amount of 4393 studies and took from March 2014 until July 2014. On the first draft, based on the checklists, the remaining 420 studies were evaluated with their full text, leaving 16 studies, which met all our inclusion criteria. Since the wide heterogeneity of the citations it was not possible for us to conduct a meta-analysis therefore we continued the study as a systematic review. Table 1 shows that the individual studies differ in results. In the discussion we were able to define the pros and cons for why this occurs, through detailed analyses.

Figure 1 displays each risk factor in a hierarchical order. The three most important risk factors seem to be male patients, an elevated BMI and a prolonged duration of operation.

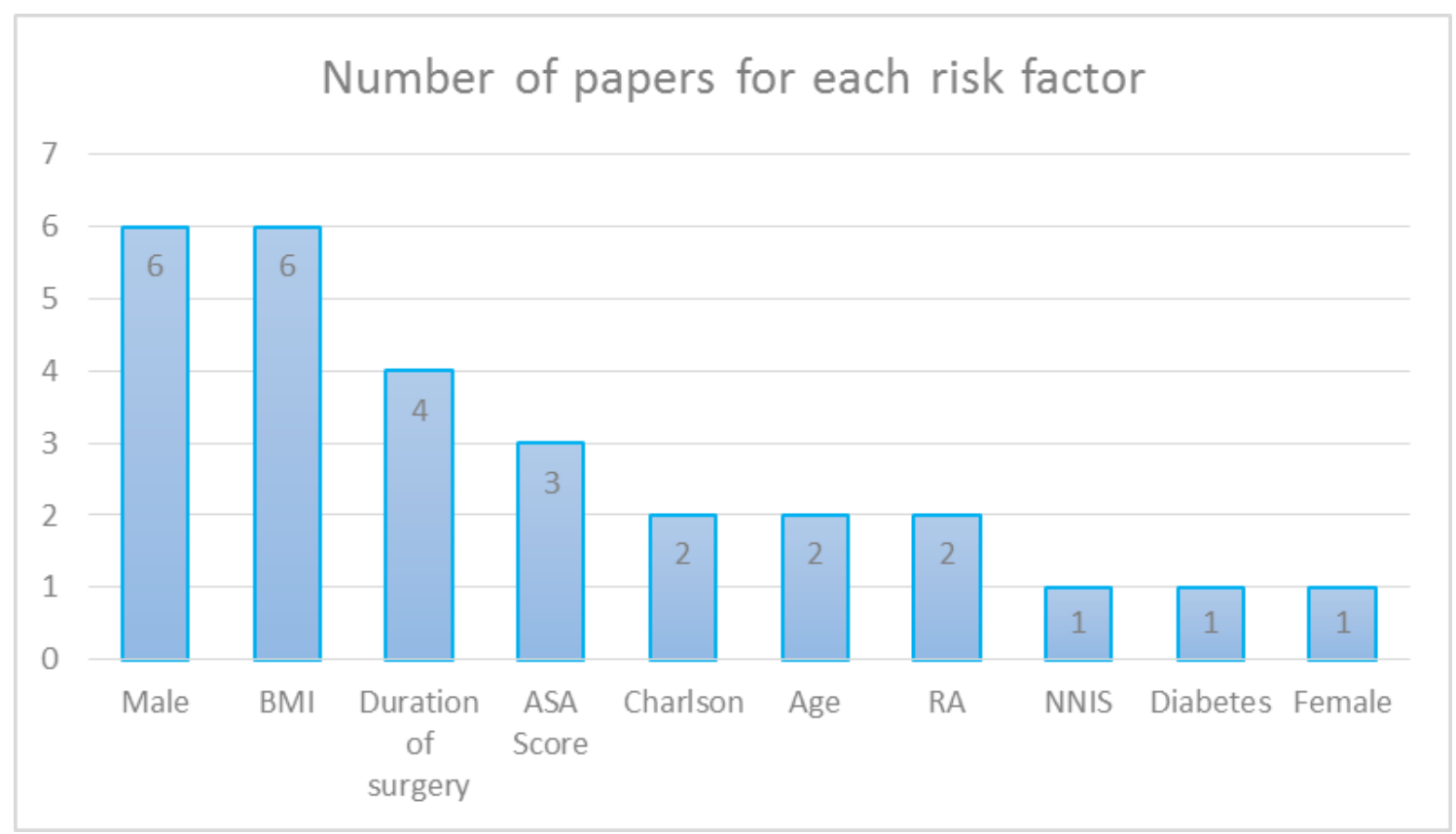

Figure 1. The risk factors are shown in a hierarchical order. The numbers display the number of studies, in which the risk factors were shown as a significant risk factor for PJI.[1-15] 


\subsection{BMI}

Six studies reported a BMI above 30 as a risk factor for developing a PJI. [4-10]

Lubbeke et al. (2007) reported a Risk Ratio of 4.4 (95\% Confidence interval: 1.8-10.8) and a Hazard Ratio of 5.1 (95\% CI: 1.9-13.5) for a BMI >30. Bozic et al. (2012) reported a Risk Ratio of 1.97 (95\% CI: 1.47-2.48), Font-Vizcarra et al. (2011) an Odds Ratio of 5.35 (95\% CI: 1.45-19.81) and Namba et al. (2012) a Hazard Ratio of 2.37 (95\% CI: $1.55-3.61)$ with a BMI $>35$. Three studies did not find any connection between an elevated BMI and an increased risk of PJI. [11-14] Namba et al. (2012) also showed that a BMI below 18.5 could be a risk factor too. [8]

\subsection{Sex}

Six studies reported an increased risk for men. [5, 9, 15-18] Dale et al. (2012) reported a Risk Ratio of 1.9 (95\% CI: 1.8-2.1) in one study and in another one (2009) a Risk Ratio of 2.5 (95\% CI: 2.1-2.9) of men. These results were confirmed by Pedersen et al. (2010) with a Risk Ratio of 1.53 (95\% CI: 1.30-1.80) and Reina et al. (2013) with an Odds Ratio of 2.3 (95\% CI: 1.7-3.8). Only Namba et al. (2012) showed an inferior risk for men with a Hazard Ratio of 0.70 (95\% CI: 0.49-0.99). Bozic et al. (2014) reported a decreased Hazard Ratio of 0.55 (95\% CI: 0.30-0.99) for women and Lubbeke et al. (2007) showed that women with a BMI above 30 are more common to suffer from a periprosthetic joint infection than men with the same BMI. $[5,7][8]$

\subsection{Duration of operation}

Four studies showed that a prolonged operation time above 100 or 120 minutes lead to an increased risk for a PJI. [16-20] Dale et al. showed that patients with an operation time between 100 to 129 minutes have a Risk Ratio of 1.3 (95\% CI: 1.0-1.5) and above 130 minutes a Risk Ratio of 1.5 (95\% CI: 1.2-1.9). Pedersen et al. (2010) reported a Risk Ratio of 2.02 (95\% CI: 1.49-2.75) by operations with a duration of 120 minutes and Ong et al. (2009) reported an Odds Ratio of 1.78 (95\% CI: 1.40-2.26) by operations that exceed 210 minutes. Only Namba et al. (2012) showed, that the operation time did not influence the outcome on the infection rate, however in this study the operation time is missing on $18 \%$ of the patients. [8]

\subsection{Diabetes}

With a relative risk of 1.45 (95\% CI: 1.00-2.09) Pedersen et al. (2010) showed, that patients with type 2 diabetes tend to develop a PJI. [21] In the first analysis Namba et al. (2012) reported a higher incidence for patients with diabetes $(0.79 \%)$ than patients without diabetes $(0.45 \%)$. These results however could not be confirmed with the multi-variant analysis. [8]

\subsection{Age}

Font-Vizcarra et al. (2011) showed in a prospective study with 428 patients, that an age above 67 years led to a higher risk for infection (Odds Ratio 5.35; 95\% CI: 1.45-19.8). Muilwijk et al. (2006) showed as well, that patients above 75 years had an odds ratio of 2.5 (CI 95\%: 0.8-8.0) and patients between 65 and 74 had an odds ratio of 2.1 (2.1 CI 95\%: 0.6-6.7). However three other studies showed, that the age had no influence for patients in developing a deep periprosthetic infection. $[5,8,13,16,19,22]$

\subsection{ASA Score}

Patients with an ASA score of 3 and above had a higher risk for periprosthetic joint infections than patients with a lower score. $[8,9,19]$

\subsection{NNIS risk index score}

Muilwijk et al. (2006) showed for patients with a „National Nosocomial Infections Surveillance System risk index score"(NNIS) from 1 an Odds Ratio of 1.4 (95\% CI: 1.0-1.9) and for patients with a NNIS of 2 an Odds Ratio of 3.1 (95\% CI: 1.9-5.1) for developing a periprosthetic joint infection. [19]

\subsection{Charlson Index}

Patients with a Charlson Index Score from 5 or above had in a study conducted by Ong et al. (2009) a 157\% higher chance to suffer from a PJI than patients with an score of 0 (Odds Ratio 2.57, 95\% CI: 1.96-3.37). Pedersen et al. (2010) showed that a Charlson Index Score from 1 or above led to a Risk Ratio from 1.30 (95\% CI: 1.09-1.54). [17, 18]

\subsection{Rheumatoid arthritis}

Two studies showed a higher risk for patients with rheumatoid arthritis. In the study from Dale et al. (2012) patients with rheumatoid arthritis had a relative risk of 1.4 (95\% CI: 1.1-1.7) and in the study from Bozic et al. (2012) they had a relative risk of 1.79 (95\% CI: 1.51-2.12). [4, 15] However three other studies could not find any relation between rheumatoid arthritis and developing a PJI. [5, 16, 18]

\subsection{Steroids}

While Kaspar et al. (2005) reported of a higher infection rate of $8 \%$ with patients getting steroid injections preoperatively, Meermans et al. (2012) showed that it is safe to treat patients preoperatively with steroids. [23, 24]

\subsection{HIV}

Graham et al. (2014) reported of none correlation between having HIV and suffering from a periprosthetic joint infection.[25]

\subsection{Bisphosphonate}

The intake of bisphosphonate postoperatively showed an 
elevated relative risk of 2.59 (95\% CI: 1.30-6.53) for developing a PJI. [26]

\section{Discussion}

\subsection{BMI}

We asked us why an elevated BMI score can lead to an increased risk of PJI. One possible explanation could lay in the inflammatory reaction. Motaghedi et al (2014) reported an increased postoperative rate of interleukin (IL) $1 \beta$ and 6 as well as the tumor necrosis factor $\alpha$ (TNF) in obese patients after a THA. These inflammatory factors are produced in the white fat cells and trigger neutrophil granulocytes which lead to an inflammatory reaction. $[27,28]$

Another study suggests that in obese patients the antibiotic concentration in the fat tissue is not sufficient. The cefazolin concentration showed to be too low in obese patients to be in the therapeutic dose against gram-negative cocci. [29]

The oxygenation of the subcutaneous fat tissue seems to take a big part in the infection rate as well. Fleischmann et al. (2005) reported a decrease rate of oxygenation in obese patients. Usually the germs would be destroyed through an oxidative process in the neutrophil granulocytes. Therefore the wound healing and the defense against infections is impaired in obese patients. [30]

Another factor could be the malnutrition. Obese patients tend to low albumin and transferrin serum levels through a high caloric and low-nutrient diet. In the study from Huang et al. (2013) $42.9 \%$ der patients who suffered from malnutrition had an elevated BMI score. The complication rates in this patient population raised to $12 \%$ while the rates in the control group stayed at $2.9 \%$. Patients with the preoperative low serum levels of transferrin suffered the most from a delayed and impaired wound healing. Jaberi et al. (2008) showed furthermore that debridement in patients with malnutrition lead to a poorer outcome than in patients with a balanced diet. [31-33]

\subsection{Sex}

Six out of seven studies reported a higher infection risk for men. In this studies however the difference between the genders were examined secondarily. None of the studies report any causes for the different infection rates. Only Pedersen and Svendsson (2010) suggest that the operation trauma in men is higher and that men are more likely to be operated, even with more and worse comorbidities than women. The explanation with the higher operation trauma seems odd because women have a genetic predisposition for accumulating fat in the hip region and therefore the trauma in women should be bigger than in men. Lubbeke et al. (2007), who investigated the difference between obese men and obese women, assume that the body fat distribution and metabolic reactions leads to a higher infection risk for obese women. $[7,18,34]$
Another cause could be an enzyme that oxidases the arachidonic acid to leukotrienes in immunocompetent cells, the 5-lipoxygenase (5-LO). Pergola et al. (2011) reported 1.8-times higher rates of 5-LO in women than in men, which leads to a better immune reaction. Testosterone seems to lower the rates and production of leukotrienes. With an injection of $5 \alpha$-dihydrotestosterone in female cells the synthesis rates of the leukotrienes decreased to the level of male cells. [35]

The examined studies show a higher risk for PJI for men. More studies should be conducted, concentrating more on the sex of the patients, to show the reasons behind the disparity in infection rates. Our results show that the sex in most cases was only examined secondarily.

\subsection{Duration of operation}

Five out of six studies showed a correlation between a prolonged operation time and a higher risk for periprosthetic joint infections. The causes can be diverse and hard to find. Patients with a higher BMI score often have a longer operation time due to larger operation areas and bigger tissue traumas. A prolonged operation time can also be a sign of a difficult operation, a slow working surgeon, an inexperienced operation team or even a combination of these factors. Hospitals with a higher rate of hip arthroplasties show a decreased operation time of 25 to 35 minutes, due to well-practiced operation teams and standardized procedures. Part of them are preoperative planning, a wide selection of prostheses for different situations, the right choice of operation technique and surgical instruments, the right position of the patient und regular training schedules for the team. $[36,37]$

Our results show that a prolonged operation time correlates with the rates of PJIs after THAs. However the operation time is multimodal factor which strongly depends on the different patients and surgeons with their team.

\subsection{Diabetes}

Three out of four studies show a higher risk of infection for patients with diabetes. Only Pedersen and Menert (2010) divided the patients in type I and type II and showed that only patients with type II diabetes tend to develop a PJI. The other studies did not divide the patients and are therefore not as viable. Pedersen and Menert (2010) conducted a comprehensible study with no flaw from our side. [21]

The pathomechanism in diabetics is varied and manifold. Through macrovascular implications the periphery vessels are damaged and the wound healing is impaired. Furthermore diabetic patients have an inferior innate immune response and function of the neutrophil granulocytes. The hyperglycemic blood levels inhibit the glucose-6-phosphatdehydrogenase in neutrophil granulocytes which leads to a higher rate of apoptosis and a lower ability of endothelium migration. Through a lower production rate of interleukin 1 and 6 in lymphocytes and 
monocytes in diabetic patients, the immune reaction is impaired. Furthermore the hyperglycemia inhibits the production of interleukin 10 in myeloid cells, as well as interferon gamma and the tumor necrosis factor alpha in T-cells. The expression of the major histocompability complex (MHC) seems also reduced in patients with hyperglycemia, which leads to a higher resistance in bacteria. $[38,39]$

Our results show a correlation between patients with diabetes type II and a higher risk for PJIs after THA. Are the glucose blood levels of patients under control and under $200 \mathrm{mg} / \mathrm{dl}$ postoperative the risk of infection decreases heavily. Furthermore the monitoring of the $\mathrm{HbAlc}$ and reduction below $8 \%$ seems very important for a further reduction of infections. [40-43]

\subsection{Age}

Most of the studies show that the THA even in old patients is still a very safe and efficient operation. Our results indicate that there are no correlations between age and infection rates.

\subsection{Rheumatoid arthritis}

The pathogenesis of the rheumatoid arthritis is still not fully clarified. The enzyme which produces the "Tyrosin-Phosphatase nonreceptor 22" (PTPN22) seems to be the cause. It leads to an altered immune path way and the typical signs of arthritis (heat, pain, redness and swelling). It seems that infection with a variety of germs (e.g.: Epstein-Barr virus) can trigger this disease. [44]

Dale and Fenstad (2012) found a significant correlation in the "NARA" register between patients with rheumatoid arthritis and an increased rate of PJI but Dale and Hallan (2009) did not see any correlation a few years earlier in the Norwegian register. There seem to be wide regional differences which are hence not explained. The same thing happened in two studies from Bozic et al. While the older study with the "Medicare" population suggests the rheumatoid arthritis as a risk factor for PJIs, the newer study showed no sign of it. Viable information, like the time of active episode and treatment is missing in the studies. More studies should be conducted with the sole attention on patients with rheumatoid arthritis to provide verified results. $[4,5,15,16]$

\section{Limitations}

The inclusion of studies that were written in either German or English can bias our results because of regional differences. The heterogeneity of the studies did not allow us to conduct a meta-analysis, which reduced the statistical significance.

\section{REFERENCES}

[1] Pivec, R., et al., Hip arthroplasty. Lancet, 2012. 380(9855): p. 1768-77.

[2] Bozic, K.J. and M.D. Ries, The impact of infection after total hip arthroplasty on hospital and surgeon resource utilization. J Bone Joint Surg Am, 2005. 87(8): p. 1746-51.

[3] Haenle, M., et al., [Economic impact of infected total hip arthroplasty in the German diagnosis-related groups system]. Orthopade, 2012. 41(6): p. 467-76.

[4] Bozic, K.J., et al., Patient-related risk factors for periprosthetic joint infection and postoperative mortality following total hip arthroplasty in Medicare patients. J Bone Joint Surg Am, 2012. 94(9): p. 794-800.

[5] Bozic, K.J., et al., Risk factors for periprosthetic joint infection following primary total hip arthroplasty: a case control study. J Arthroplasty, 2014. 29(1): p. 154-6.

[6] Font-Vizcarra, L., et al., Relationship between intraoperative cultures during hip arthroplasty, obesity, and the risk of early prosthetic joint infection: a prospective study of 428 patients. Int J Artif Organs, 2011. 34(9): p. 870-5.

[7] Lubbeke, A., et al., Differences in outcomes of obese women and men undergoing primary total hip arthroplasty. Arthritis Rheum, 2007. 57(2): p. 327-34.

[8] Namba, R.S., M.C. Inacio, and E.W. Paxton, Risk factors associated with surgical site infection in 30,491 primary total hip replacements. J Bone Joint Surg Br, 2012. 94(10): p. 1330-8.

[9] Reina, N., et al., Infection as a cause of primary total hip arthroplasty revision and its predictive factors. Orthop Traumatol Surg Res, 2013. 99(5): p. 555-61.

[10] Renaud, A., M. Lavigne, and P.A. Vendittoli, Periprosthetic joint infections at a teaching hospital in 1990-2007 - Tabelle. Can J Surg, 2012. 55(6): p. 396.

[11] Andrew, J.G., et al., Obesity in total hip replacement. J Bone Joint Surg Br, 2008. 90(4): p. 424-9.

[12] Davis, A.M., et al., Does body mass index affect clinical outcome post-operatively and at five years after primary unilateral total hip replacement performed for osteoarthritis? A multivariate analysis of prospective data. J Bone Joint Surg Br, 2011. 93(9): p. 1178-82.

[13] Malinzak, R.A., et al., Morbidly obese, diabetic, younger, and unilateral joint arthroplasty patients have elevated total joint arthroplasty infection rates. J Arthroplasty, 2009. 24(6 Suppl): p. 84-8.

[14] Moran, M., et al., Does body mass index affect the early outcome of primary total hip arthroplasty? J Arthroplasty, 2005. 20(7): p. 866-9.

[15] Dale, H., et al., Increasing risk of prosthetic joint infection after total hip arthroplasty. Acta Orthop, 2012. 83(5): p. 449-58.

[16] Dale, H., et al., Increasing risk of revision due to deep infection after hip arthroplasty. Acta Orthop, 2009. 80(6): p. 639-45.

[17] Ong, K.L., et al., Prosthetic joint infection risk after total hip 
arthroplasty in the Medicare population. J Arthroplasty, 2009. 24(6 Suppl): p. 105-9.

[18] Pedersen, A.B., et al., Risk factors for revision due to infection after primary total hip arthroplasty. A population-based study of 80,756 primary procedures in the Danish Hip Arthroplasty Registry. Acta Orthop, 2010. 81(5): p. 542-7.

[19] Muilwijk, J., et al., Random effect modelling of patient-related risk factors in orthopaedic procedures: results from the Dutch nosocomial infection surveillance network 'PREZIES'. J Hosp Infect, 2006. 62(3): p. 319-26.

[20] Cordero-Ampuero, J. and M. de Dios, What are the risk factors for infection in hemiarthroplasties and total hip arthroplasties? Clin Orthop Relat Res, 2010. 468(12): p. 3268-77.

[21] Pedersen, A.B., et al., Risk of revision of a total hip replacement in patients with diabetes mellitus: a population-based follow up study. J Bone Joint Surg Br, 2010. 92(7): p. 929-34.

[22] Choong, P.F., et al., Risk factors associated with acute hip prosthetic joint infections and outcome of treatment with a rifampinbased regimen. Acta Orthop, 2007. 78(6): p. 755-65.

[23] Kaspar, S. and V.d.B.J. de, Infection in hip arthroplasty after previous injection of steroid. J Bone Joint Surg Br, 2005. 87(4): p. 454-7.

[24] Meermans, G., K. Corten, and J.P. Simon, Is the infection rate in primary THA increased after steroid injection? Clin Orthop Relat Res, 2012.470(11): p. 3213-9.

[25] Graham, S.M., et al., Total hip replacement in HIV-positive patients. Bone Joint J, 2014. 96-b(4): p. 462-6.

[26] Thillemann, T.M., et al., Postoperative use of bisphosphonates and risk of revision after primary total hip arthroplasty: a nationwide population-based study. Bone, 2010. 46(4): p. 946-51.

[27] Motaghedi, R., et al., Association of obesity with inflammation and pain after total hip arthroplasty. Clin Orthop Relat Res, 2014. 472(5): p. 1442-8.

[28] Eder, K., et al., The major inflammatory mediator interleukin-6 and obesity. Inflamm Res, 2009. 58(11): p. 727-36.

[29] Pevzner, L., et al., Effects of maternal obesity on tissue concentrations of prophylactic cefazolin during cesarean delivery. Obstet Gynecol, 2011. 117(4): p. 877-82.

[30] Fleischmann, E., et al., Tissue oxygenation in obese and non-obese patients during laparoscopy. Obes Surg, 2005. 15(6): p. 813-9.
[31] Huang, R., et al., The effect of malnutrition on patients undergoing elective joint arthroplasty. J Arthroplasty, 2013. 28(8 Suppl): p. 21-4.

[32] Gherini, S., et al., Delayed wound healing and nutritional deficiencies after total hip arthroplasty. Clin Orthop Relat Res, 1993(293): p. 188-95.

[33] Jaberi, F.M., et al., Procrastination of wound drainage and malnutrition affect the outcome of joint arthroplasty. Clin Orthop Relat Res, 2008. 466(6): p. 1368-71.

[34] Larsson, I., et al., Body composition in the SOS (Swedish Obese Subjects) reference study. Int J Obes Relat Metab Disord, 2004. 28(10): p. 1317-24.

[35] Pergola, C., et al., Testosterone suppresses phospholipase D, causing sex differences in leukotriene biosynthesis in human monocytes. Faseb j, 2011. 25(10): p. 3377-87.

[36] Kessler, S., et al., Influence of operation duration on perioperative morbidity in revision total hip arthroplasty. Acta Orthop Belg, 2003. 69(4): p. 328-33.

[37] Smabrekke, A., et al., Operating time and survival of primary total hip replacements: an analysis of 31,745 primary cemented and uncemented total hip replacements from local hospitals reported to the Norwegian Arthroplasty Register 1987-2001. Acta Orthop Scand, 2004. 75(5): p. 524-32.

[38] Price, C.L., et al., Methylglyoxal modulates immune responses: relevance to diabetes. J Cell Mol Med, 2010. 14(6b): p. 1806-15.

[39] Casqueiro, J., J. Casqueiro, and C. Alves, Infections in patients with diabetes mellitus: A review of pathogenesis. Indian J Endocrinol Metab, 2012. 16 Suppl 1: p. S27-36.

[40] Peleg, A.Y., et al., Common infections in diabetes: pathogenesis, management and relationship to glycaemic control. Diabetes Metab Res Rev, 2007. 23(1): p. 3-13.

[41] Deshpande, A.D., M. Harris-Hayes, and M. Schootman, Epidemiology of diabetes and diabetes-related complications. Phys Ther, 2008. 88(11): p. 1254-64.

[42] Latham, R., et al., The association of diabetes and glucose control with surgical-site infections among cardiothoracic surgery patients. Infect Control Hosp Epidemiol, 2001. 22(10): p. 607-12.

[43] Zerr, K.J., et al., Glucose control lowers the risk of wound infection in diabetics after open heart operations. Ann Thorac Surg, 1997. 63(2): p. 356-61.

[44] Tang, G.P., L. Hu, and Q.H. Zhang, [PTPN22 1858C/T polymorphism is associated with rheumatoid arthritis susceptibility in Caucasian population: a meta-analysis]. Zhejiang Da Xue Xue Bao Yi Xue Ban, 2014. 43(4): p. 466-73. 